Case report

\title{
Post-thrombolysis hemorrhage in a patient with hypothyroidism and acute ischemic stroke: Case report
}

\author{
Furong $\mathrm{Gu}$, Jie Qin, Rongjie Chen, Xiaolin $\mathrm{Xu}$ * \\ Neurology Department, Tianjin Huanhu Hospital, Tianjin, China
}

\section{A R T I C L E I N F O}

Article history:

Received 26 March 2015

Accepted 5 May 2015

Available online 18 May 2015

\section{Keywords:}

Hypothyroidism

Thrombolysis

Recombinant tissue plasminogen

activator

Acute ischemic stroke

Edema

\begin{abstract}
A B S T R A C T
Thrombolytic treatment with intravenous recombinant tissue plasminogen activator (rtPA) is an effective treatment for acute ischemic stroke. However, its effectiveness and risks in patients with hypothyroidism have not been reported. Here, we report the case of hemorrhagic transformation after intravenous rtPA thrombolysis treatment in a patient with acute ischemic stroke and hypothyroidism. An apparent edema formed around the hematoma and progressively worsened. He also developed lung infection, electrolyte imbalance, and abnormal liver and kidney functions, and eventually died within 1 month of symptom onset. Thus, our observations suggest that caution should be exercised for the administration of intravenous rtPA thrombolysis to patients with hypothyroidism.
\end{abstract}

(C) 2015 Polish Neurological Society. Published by Elsevier Sp. z o.o. All rights reserved.

\section{Introduction}

Hypothyroidism, a common endocrine and metabolic disorder, is a clinical syndrome caused by the insufficiency in the synthesis, secretion, or biological efficacy of the thyroid hormone [1]. Hypothyroidism accelerates atherosclerosis and increases the incidence of atherosclerotic ischemic stroke [2]. Thrombolytic treatment with intravenous recombinant tissue plasminogen activator (rtPA) is an effective treatment for acute ischemic stroke [3]. However, its effectiveness and risks in patients with hypothyroidism have not been reported. We report a patient with acute ischemic stroke and hypothyroidism who died of hemorrhagic transformation after receiving intravenous rtPA thrombolysis treatment.

\section{Case report}

A 53-year-old right-handed male patient was hospitalized $1.5 \mathrm{~h}$ after experiencing sudden slurred speech and muscle weakness in the right extremities. He had a 5-year history of hypertension and 3-year history of hypothyroidism, and had been taking $50 \mu \mathrm{g}$ qd of levothyroxine sodium tablets. On admission, the patient was conscious, and showed dysarthria, right central facial paralysis, 1/5 myodynamia in the right upper extremity, 0/5 myodynamia in the right lower extremity,

\footnotetext{
* Corresponding author at: Neurology Department, Tianjin Huanhu Hospital, Qixiangtai Road 122, 300060, Tianjin, China. Tel.: +86 22 60367898; fax: +8622 60367651.

E-mail address: hhyyxxl@163.com (X.Xu).

http://dx.doi.org/10.1016/j.pjnns.2015.05.002

0028-3843/@ 2015 Polish Neurological Society. Published by Elsevier Sp. z o.o. All rights reserved.
} 


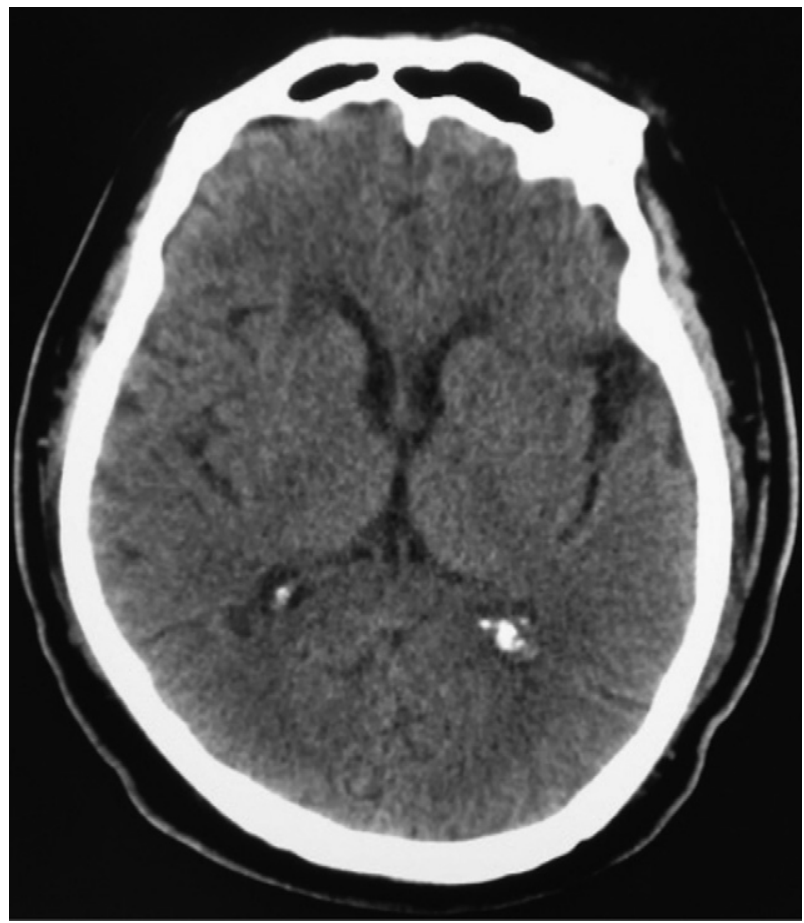

Fig. 1 - Cranial computed tomography before recombinant tissue plasminogen activator thrombolysis shows no hemorrhage.

$5 / 5$ myodynamia in the left limbs, hypalgesia in the right side of the body, and blood pressure of $170 / 90 \mathrm{mmHg}$. Cranial computed tomography (CT) taken $1.5 \mathrm{~h}$ after onset did not show any abnormalities (Fig. 1). Routine blood test, coagulation function, and liver and kidney functions were all normal. A random blood glucose level of $8 \mathrm{mmol} / \mathrm{L}$ was obtained, and the patient's National Institute of Health Stroke Scale (NIHSS) score was 12. Thus, the patient was treated with $70 \mathrm{mg}$ intravenous rtPA thrombolysis $3 \mathrm{~h}$ after the onset based on a dosage of $0.6 \mathrm{mg} / \mathrm{kg}$ body weight (patient's body weight $115 \mathrm{~kg}$ ), in addition to intravenous urapidil antihypertensive therapy. Upon completion of the rtPA thrombolysis treatment, the patient regained consciousness, but showed dysarthria, right central facial paralysis, $2 / 5$ myodynamia in the right upper extremity, 1/5 myodynamia in the right lower extremity, and $5 / 5$ myodynamia in the left limbs. One hour after rtPA thrombolysis treatment, the patient experienced headache, vomiting, and drowsiness. Cranial CT reexamination revealed hemorrhage in the right temporal lobe extending to the outer right side, a parenchymal hematoma approximate $3.5 \mathrm{~cm} \times 2 \mathrm{~cm} \times 4 \mathrm{~cm}$ in size, and a spotted hemorrhage in the left basal ganglia (Fig. 2). Cranial CT reexamination $24 \mathrm{~h}$ later showed no increase in bleeding, an apparent compression of the right ventricles, a slight left shift of the midline, and a left basal ganglia infarction with hemorrhage (Fig. 3). The patient was lethargic, opened his eyes on stimulation, presented occasional spontaneous movement of the left limbs, and did not respond to stimulation on the right limbs. Thyroid function test results were all significantly below normal levels as follows: thyroxine $\left(\mathrm{T}_{4}\right), 26.4 \mathrm{nmol} / \mathrm{L}(66-181 \mathrm{nmol} / \mathrm{L})$; free

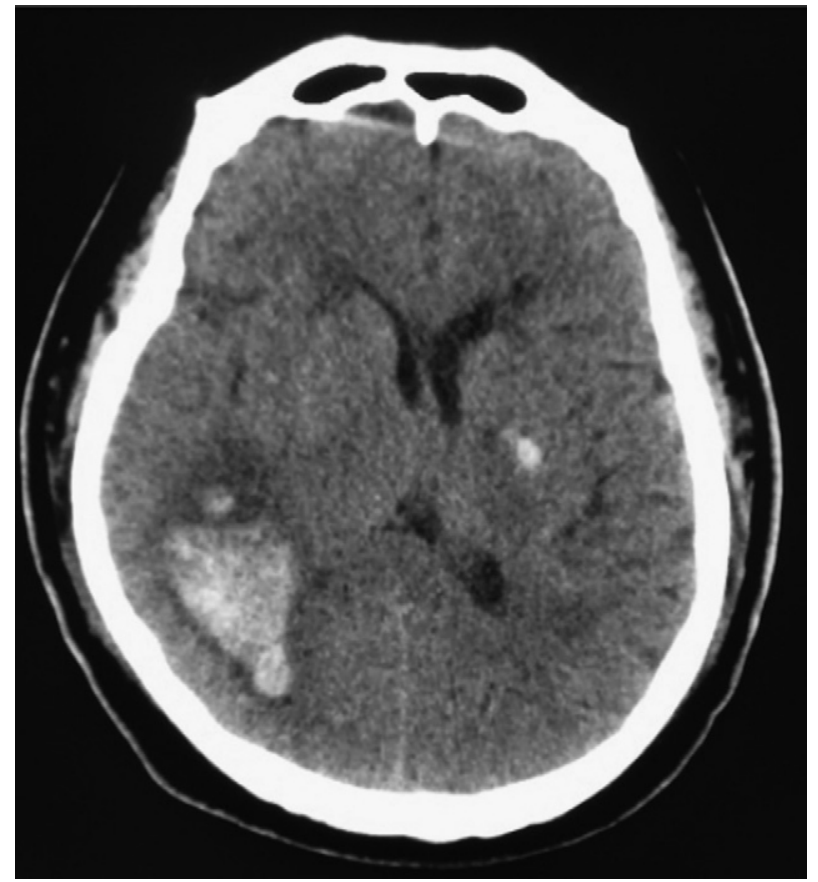

Fig. 2 - Cranial CT $1 \mathrm{~h}$ after rtPA thrombolysis shows hemorrhage in the right temporal lobe and spotted hemorrhage in the left basal ganglia.

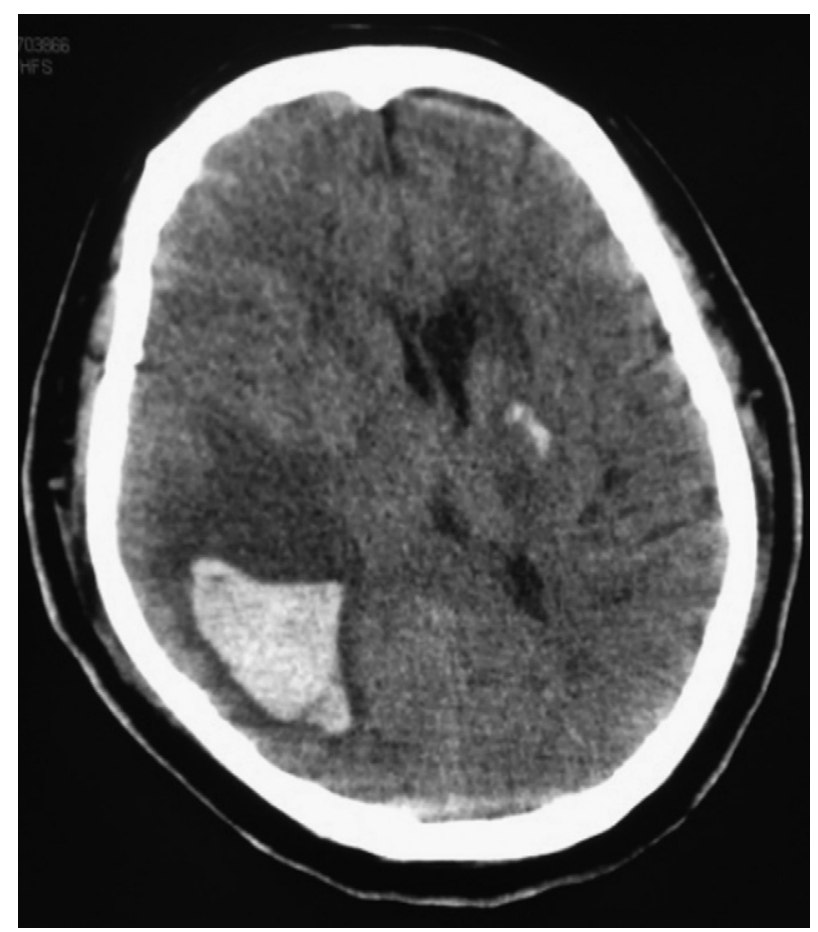

Fig. 3 - Cranial CT $24 \mathrm{~h}$ after rtPA thrombolysis shows hemorrhage in the right temporal lobe surrounded by edema, compressed right ventricle, infarction, and spotted hemorrhage in the left basal ganglia. 
thyroxine $\left(\mathrm{FT}_{4}\right) 3.47 \mathrm{pmol} / \mathrm{L}(12-22 \mathrm{pmol} / \mathrm{L})$; triiodothyronine ( $\left.\mathrm{T}_{3}\right) \quad 0.42 \mathrm{nmol} / \mathrm{L}(1.3-3.1 \mathrm{nmol} / \mathrm{L})$; and free triiodothyronine $\left(\mathrm{FT}_{3}\right) 1.54 \mathrm{pmol} / \mathrm{L}$ (3.1-6.8 pmol/L). Thyroid-stimulating hormone (TSH) $65.7 \mathrm{mIU} / \mathrm{L} \quad(0.27-4.2 \mathrm{mIU} / \mathrm{L})$ and anti-thyroid peroxidase antibody (ATPO) $252.1 \mathrm{IU} / \mathrm{mL}$ (0-34 IU/mL) levels were significantly above normal levels. Considering primary hypothyroidism of the patient, oral administration of levothyroxine sodium tablet was administered in gradually increased dosage. As the patient showed apparent intracranial edema, a combination of mannitol, albumin, and blood plasma drip was given to reduce the symptom. Cranial CT reexamination 3 days later showed no increase in cerebral bleeding, apparent edema, and compression of the right ventricle (Fig. 4). Cranial CT reexamination 14 days later showed that the blood was almost absorbed, whereas the apparent edema, compression of the right ventricle, and left shift of the midline persisted (Fig. 5). Thyroid function re-examination showed a gradual increase in $\mathrm{T}_{4}$ and $\mathrm{FT}_{4}$ levels to almost normal levels, a decrease in TSH level, and no noticeable change in $\mathrm{T}_{3}$ and $\mathrm{FT}_{3}$ levels. The patient constantly appeared lethargic, and gradually developed a lung infection, electrolyte imbalance, and abnormal liver and kidney function. The patient voluntarily discharged after 22 days of hospitalization. On discharge, the patient lost consciousness, occasionally opened his eyes, and had an automatic respiratory rhythm of 17 breaths/min and a heart rate of 100 beats/min. He died 1 day later.

\section{Discussion}

The patient in our case had no contraindication for rtPA thrombolysis, and was treated with intravenous rtPA

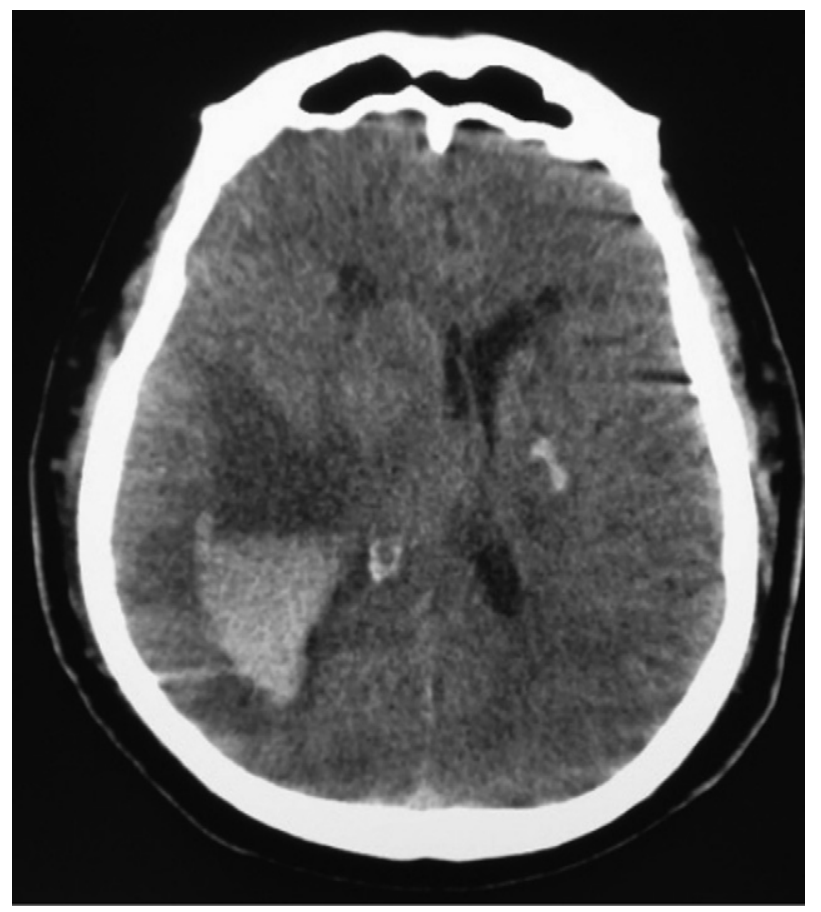

Fig. 4 - Cranial CT 3 days after rtPA thrombolysis shows no increase in bleeding but apparent edema, compressed right ventricle, and left shifted midline.

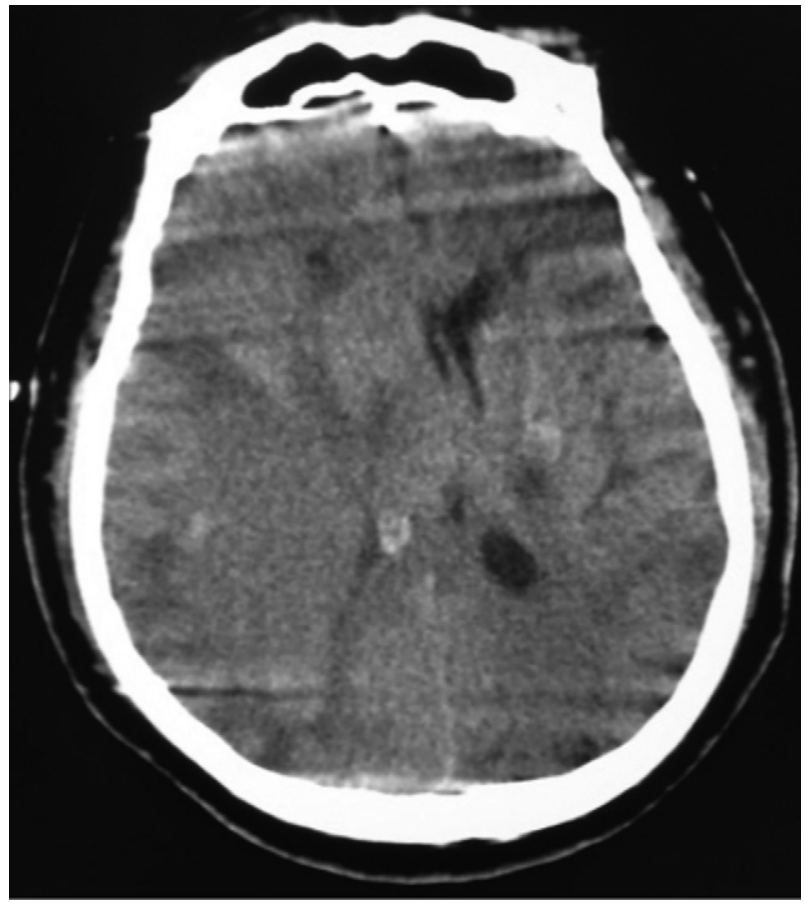

Fig. 5 - Cranial CT 14 days after rtPA thrombolysis shows that hematoma is absorbed. Apparent edema, compressed right ventricle, and left shifted midline are still visible.

thrombolysis $3 \mathrm{~h}$ after acute ischemic stroke onset. Blood tests performed before rtPA thrombolysis were unremarkable. However, after rtPA thrombolysis, hemorrhagic transformation occurred. Incidence of hemorrhagic transformation after rtPA thrombolysis administered within $3 \mathrm{~h}$ of ischemic stroke is about $6 \%$ [4], occurring mainly due to coagulation disorder and damage of the blood-brain barrier caused by rtPA. Factors associated with hemorrhagic transformation include advanced age, serious stroke at baseline, large area of baseline infarction, hyperglycemia, hypertension, a history of antiplatelet drug use, atrial fibrillation, congestive heart failure, and impaired renal function [4]. The patient had a normal blood glucose level, and his controlled pre-thrombolysis blood pressure was within the recommended range. He had no other risk factors and the dosage of rtPA $(0.6 \mathrm{mg} / \mathrm{kg})$ was low; however, hemorrhage still occurred. Thus, further investigation was needed to explore the cause of hemorrhagic transformation. Vascular lesions form and progressively worsen in the early stage of cerebral focal ischemia [5]. This can damage the blood-brain barrier and increase the risk of hemorrhagic transformation. Collateral circulation could reduce the damage to the ischemic vessels, and thus, perfusion status has been closely related to hemorrhagic transformation after revascularization [6,7]. Research has shown that patients with poor collateral circulation have a higher risk of hemorrhage after recanalization of blood vessels [8]. It is known that hypothyroidism increases the incidence of risk factors associated with atherosclerosis, such as hypertension, hyperlipidemia, endothelial dysfunction, hyperhomocysteinemia, and elevated hypersensitive C-reactive protein level, which can further accelerate the progress of atheroscle- 
rosis $[2,9]$. It is speculated that hypothyroidism in our patient caused extensive atherosclerosis, poor collateral circulation, poor perfusion status, and elevated risk of hemorrhage after recanalization of blood vessels. The patient exhibited left basal ganglia infarction and the offending vessels were the perforating branches of the middle cerebral artery. Recanalization of vessels occurred after rtPA thrombolysis and hemorrhage occurred at the left basal ganglia. Since atherosclerosis is a risk factor for intracranial hemorrhage, the hemorrhage at the right temporal lobe of the patient may be related to significant atherosclerosis, damaged blood vessels after infusion of rtPA, and damage to the blood-brain barrier. Because the patient had none of the risk factors that could increase hemorrhage, and the rtPA dose was low, it was necessary to consider extensive atherosclerosis caused by hypothyroidism as a cause for hemorrhage.

The actual hemorrhage was about $14 \mathrm{~mL}$ after hemorrhagic transformation, but the edema was apparent. Application of mannitol, albumin, and blood plasma drips did not effectively alleviate the edema. Related studies show that hemorrhage after rtPA thrombolysis may cause milder edema compared with that in spontaneous intracerebral hemorrhage [10]. The prognosis of an intracerebral hemorrhage is directly related to the amount of hemorrhage, and patients who have less than $25 \mathrm{~mL}$ of hemorrhage tend to have a better prognosis [11]. This patient had $14 \mathrm{~mL}$ of hemorrhage, but had persistent edema, and eventually died. In the case of hypothyroidism, glycosaminoglycan accumulates under vascular endothelia, while the capillary walls become less efficient as a barrier, causing plasma protein and serum to leak into the interstitial spaces, and result in edema. The mechanism of edema formation after intracerebral hemorrhage is vasogenic. Because of the increased microvascular permeability, protein-containing fluid enters the interstitial spaces and leads to edema. In our patient, hypothyroidism increased the permeability of the capillary walls and consequent damage to the blood-brain barrier. This increased the amount of protein-containing fluid entering the interstitial spaces, thus causing the vasogenic edema to deteriorate. Consequently, an apparent edema with minor hemorrhage developed. Dehydrant did not have a significant effect and the edema was still significant after the hematomas were absorbed. The patient eventually died.

Hypothyroidism is a risk factor for atherosclerosis, and accelerates the progress of atherosclerosis and increases the risk of atherosclerotic ischemic stroke. Incidence of ischemic stroke in patients with hypothyroidism is higher than that in the general population, although patients with hypothyroidism have better prognoses after ischemic stroke. Research reveals that after a transient ischemic attack, hypothyroidism does not protect the hippocampal neurons but delays their death [12]. Due to the pre-tolerance of hypoxia and reduced sensitivity to physical stress in patients with subclinical hypothyroidism, the prognosis of these patients after infarction are better than that in patients without hypothyroidism $[13,14]$. Similarly, the prognosis may also be better in patients with hypothyroidism. However, these patients have a higher risk of hemorrhage after rtPA thrombolysis and develop significant edema, which affects prognosis. These indicate that hypothyroidism should be considered as a relative contraindication to intravenous rtPA thrombolysis therapy, or that the choice of rtPA thrombolysis for the treatment of patients with both hypothyroidism and acute ischemic stroke should be made with great caution.

The non-thyroidal illness syndrome (NTIS; or 'euthyroid sick syndrome', or low- $\mathrm{T}_{3}$ syndrome) describes abnormalities in thyroid function tests in the absence of obvious thyroid disease [15]. The most common abnormality is a decrease in $\mathrm{T}_{3}$ levels; in the presence of more severe illness, a fall in the levels of $\mathrm{T}_{4}$ occurs while the TSH levels do not elevate. Low $\mathrm{T}_{3}$ is a strong predictor of outcome in acute stroke patients [16]. In this case, TSH level was significantly above normal level and the patient presented hypothyroidism, it was different from this syndrome. However, the low $\mathrm{T}_{3}$ level is also related to poor prognosis in this case.

In this case, the patient had both hypothyroidism and acute ischemic stroke, and hemorrhagic transformation and severe edema occurred after low-dose rtPA thrombolysis. Because of the critical condition of the patient, cerebral magnetic resonance imaging and vascular examinations were not taken. Based on this case, it is suggested that the rtPA thrombolysis treatment should be considered with great care for patients with acute ischemic stroke and hypothyroidism. However, this was based on the data from one patient, and accumulation of cases and related basic research studies are needed to be conclusive and provide better treatment options to patients with hypothyroidism and acute ischemic stroke.

\section{Conflict of interest}

None declared.

\section{Acknowledgement and financial support}

None declared.

\section{Ethics}

The work described in this article has been carried out in accordance with The Code of Ethics of the World Medical Association (Declaration of Helsinki) for experiments involving humans; Uniform Requirements for manuscripts submitted to Biomedical journals.

\section{Appendix A. Supplementary data}

Supplementary data associated with this article can be found, in the online version, at doi:10.1016/j.pjnns.2015.05.002.

\section{R E F E R E N C E S}

[1] McDermott MT. In the clinic. Hypothyroidism. Ann Intern Med 2009;151(11):ITC61. 
[2] Squizzato A, Gerdes VE, Brandjes DP, Buller HR, Stam J. Thyroid diseases and cerebrovascular disease. Stroke 2005;36(10):2302-10.

[3] Jauch EC, Saver JL, Adams Jr HP. Guidelines for the early management of patients with acute ischemic stroke. Stroke 2013;44:870-947.

[4] Whiteley WN, Slot HB, Fernandes P, Sandercock P, Wardlaw J. Risk factors for intracranial hemorrhage in acute ischemic stroke patients treated with recombinant tissue plasminogen activator: a systematic review and metaanalysis of 55 studies. Stroke 2012;43(11):2904-9.

[5] Cipolla MJ, McCall AL, Lessov N, Porter JM. Reperfusion decreases myogenic reactivity and alters middle cerebral artery function after focal cerebral ischemia in rats. Stroke 1997;28(1):176-80.

[6] Kim JH, Bang OY, Liebeskind DS, Ovbiagele B, Kim GM, Chung CS, et al. Impact of baseline tissue status (diffusionweighted imaging lesion) versus perfusion status (severity of hypoperfusion) on hemorrhagic transformation. Stroke 2010;41(3):e135-42.

[7] Campbell BC, Christensen S, Butcher KS, Gordon I, Parsons MW, Desmond PM, et al. Regional very low cerebral blood volume predicts hemorrhagic transformation better than diffusion-weighted imaging volume and thresholded apparent diffusion coefficient in acute ischemic stroke. Stroke 2010;41(1):82-8.

[8] Bang OY, Saver JL, Kim SJ, Kim GM, Chung CS, Ovbiagele B, et al. Collateral flow averts hemorrhagic transformation after endovascular therapy for acute ischemic stroke. Stroke 2011;42(8):2235-9.

[9] Ichiki T. Thyroid hormone and atherosclerosis. Vascul Pharmacol 2010;52(3-4):151-6.
[10] Gebel JM, Brott TG, Sila CA, Tomsick TA, Jauch E, Salisbury $S$, et al. Decreased perihematomal edema in thrombolysisrelated intracerebral hemorrhage compared with spontaneous intracerebral hemorrhage. Stroke 2000;31 (3):596-600

[11] Christoforidis GA, Slivka A, Mohammad Y, Karakasis C, Avutu B, Yang M. Size matters: hemorrhage volume as an objective measure to define significant intracranial hemorrhage associated with thrombolysis. Stroke 2007;38 (6):1799-804

[12] Lee CH, Yoo KY, Hwang IK, Choi JH, Park OK, Li H, et al. Hypothyroid state does not protect but delays neuronal death in the hippocampal CA1 region following transient cerebral ischemia: focus on oxidative stress and gliosis. J Neurosci Res 2010;88(12):2661-8.

[13] Akhoundi FH, Ghorbani A, Soltani A, Meysamie A. Favorable functional outcomes in acute ischemic stroke patients with subclinical hypothyroidism. Neurology 2011;77(4):349-54.

[14] Baek JH, Chung PW, Kim YB, Moon HS, Suh BC, Jin DK, et al. Favorable influence of subclinical hypothyroidism on the functional outcomes in stroke patients. Endocr J 2010;57 (1):23-9.

[15] Alevizaki M, Synetou M, Xynos K, Pappa T, Vemmos KN. Low triiodothyronine: a strong predictor of outcome in acute stroke patients. Eur J Clin Invest 2007;37: 651-7.

[16] Ambrosius W, Kazmierski R, Gupta V, Warot AW, Adamczewska-Kociałkowska D, Błazejewska A, et al. Low free triiodothyronine levels are related to poor prognosis in acute ischemic stroke. Exp Clin Endocrinol Diabetes 2011;119(3):139-43. 